\title{
SCIENTIFIC REP RTS

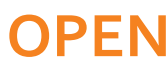 \\ Characterization of species-specific genes regulated by E2-2 in human plasmacytoid dendritic cells
}

Received: 03 February 2015

Accepted: 27 April 2015

Published: 17 July 2015

\author{
Menglan Cheng ${ }^{1}$, Xuyuan Zhang ${ }^{1}$, Haisheng $\mathrm{Yu}^{1}$, Peishuang Du ${ }^{1}$, Joël Plumas ${ }^{2}$, \\ Laurance Chaperot ${ }^{2}$, Lishan Su' ${ }^{1,3}$ \& Liguo Zhang ${ }^{1}$
}

Dendritic cells (DCs) are sentinels of the immune system and comprise two distinct subsets: conventional DCs (cDCs) and plasmacytoid DCs (pDCs). Human pDCs are distinguished from mouse pDCs phenotypically and functionally. Basic helix-loop-helix protein E2-2 is defined as an essential transcription factor for mouse PDC development, cell fate maintenance and gene programe. It is unknown whether E2-2 regulation contributes to this species-specific difference. Here we investigated the function of E2-2 in human PDCs and screened human-specific genes regulated by E2-2. Reduced E2-2 expression in human PDC cell line GEN2.2 resulted in diminished IFN- $\alpha$ production in response to $\mathrm{CpG}$ but elevated antigen presentation capacity. Gene expression profiling showed that E2-2 silence down-regulated $\mathrm{pDC}$ signature genes but up-regulated $\mathrm{CDC}$ signature genes. Thirty humanspecific genes regulated by E2-2 knockdown were identified. Among these genes, we confirmed that expression of Siglec- 6 was inhibited by E2-2. Further more, Siglec- 6 was expressed at a higher level on a human pDC subset with drastically lower expression of E2-2. Collectively, these results highlight that E2-2 modulates PDC function in a species-specific manner, which may provide insights for PDC development and functions.

Dendritic cells (DCs) are critical mediators for innate and adaptive immune responses. They are composed of two subsets: cDCs professional in antigen capture, processing and presentation, and pDCs dedicated to producing type I interferon (IFN-I) upon stimulation.

Although functional equivalences have been defined through comparative genomics ${ }^{1,2}$, there are notable discrepancies between human and mouse pDCs. Upon stimulation, mouse pDCs can produce IL-12 while human pDCs cannot ${ }^{3-5}$. Studies have suggested that, in response to LPS or CpG plus CD40L, human pDCs produce as much IL-12 as do cDCs but it's subsequently proved to be resulted from contaminating $\mathrm{cDCs}^{5}$. The expression patterns of Toll like receptors by pDCs in mouse and human are different. Mouse pDCs express most TLRs except for TLR $3^{6}$, while human pDCs only express TLR1, TLR6, and prominent TLR7 and TLR9 $9^{7,8}$. Moreover, human and mouse pDCs express distinct surface markers. Human pDCs are marked by BDCA2, BDCA4, ILT7, CD123 and CD4. Of these markers, BDCA2 displays only $50.7 \%$ sequence identity at the amino acid level to its putative murine ortholog dectin- $2^{9}$. Currently, anti-BDCA4 magnetic bead isolation kits are widely used to enrich human pDCs from blood or tissues, while mouse pDCs don't express BDCA4 and they are enriched by anti-Bst2 magnetic beads. Mouse pDCs are marked by CD11c, Siglec-H, Bst2, B220, Gr-1, Ly-6C and Ly49Q. CD11c is a cDC marker both for human and mouse but human pDCs don't express it. Mouse pDCs are uniquely characterized by highly expressed Ly49Q, a C-type lectin-like receptor, which is crucial for TLR9-mediated type I IFN and IL-12 production by mouse $\mathrm{pDCs}^{10,11}$. Besides, human pDCs express high levels of IL-3

\footnotetext{
${ }^{1}$ Key Laboratory of Immunity and Infection, Institute of Biophysics, University of Chinese Academy of Sciences, Beijing, China. 'Department of Research and Development, EFS Rh ône-Alpes Grenoble, La Tronche, France. ${ }^{3}$ Lineberger Comprehensive Cancer Center, Department of Microbiology and Immunology, School of Medicine, University of North Carolina at Chapel Hill, Chapel Hill, NC, USA. Correspondence and requests for materials should be addressed to L.Z. (email: liguozhang@sun5.ibp.ac.cn)
} 
receptor CD123 and are highly responsive to IL-3 stimulation ${ }^{12}$. However, Mouse pDCs express low levels of IL-3 receptor and do not respond to IL- $3^{13}$.

E2-2 is preferentially expressed in human and mouse pDCs, and has been identified as a key transcription factor for pDC development and cell fate maintenance ${ }^{14,15}$. Constitutive or conditional deletion of E2-2 blocks the development of mouse pDCs but not other lineages of immune cells. The function of E2-2 in human pDCs has been explored via human hematopoietic stem cells (HSCs) differentiation assay in vitro. Over expression and RNAi-mediated knockdown of E2-2 suggest a critical role of it in pDC development ${ }^{16}$. In patients of Pitt-Hopkins Syndrome (PHS $)^{14}$, which is associated with E2-2 haploinsufficiency, all major immune cell types are present in normal numbers in peripheral blood except for pDCs. In view of above differences between human and mouse pDCs, how E2-2 contributes to the species-specific differences is unclear yet.

In this report, we characterize the consequence of E2-2 reduction in a human pDC cell line GEN2.2. Function of E2-2 in human pDCs is in line with previous observation in mouse pDCs. Besides, cDNA array data indicates that E2-2 modulates the expression of some human specific genes including Siglec- 6 .

\section{Materials and Methods}

Cell Lines and Reagents. GEN2.2 cells were cultured with MS5 feeder cells in RPMI 1640 GlutaMAX (Invitrogen) supplemented with $10 \%(\mathrm{v} / \mathrm{v})$ heat-inactivated fetal bovine serum (FBS, Invitrogen), $2 \mathrm{mM}$ L-glutamine (Invitrogen), $100 \mu \mathrm{M}$ non-essential amino acid (Invitrogen), $1 \mathrm{mM}$ sodium pyruvate (Lab Amresco), $10 \mathrm{mM}$ HEPES (Amresco), $100 \mathrm{units} / \mathrm{ml}$ penicillin and $100 \mu \mathrm{g} / \mathrm{ml}$ streptomycin (Invitrogen). HEK293T cells were grown in Dulbecco's modified Eagle's medium (DMEM) supplemented with 10\% (v/v) heat-inactivated FBS (Hyclone), $2 \mathrm{mM}$ L-glutamine, $10 \mathrm{mM}$ HEPES, $100 \mathrm{units} / \mathrm{ml}$ penicillin and $100 \mu \mathrm{g} / \mathrm{ml}$ streptomycin. Mouse anti-HA (F-7) (sc-7392) antibody was from Santa Cruz Biotechnology. Rabbit polyclonal antibody to E2-2 (ab72586) was from Sigma. Rabbit polyclonal antibody against GFP was from Proteintech. Naïve $\mathrm{CD}^{+} \mathrm{T}$ cell Isolation Kit II (human) was from Miltenyi Biotec. 5(6)-Carboxyfluorescein diacetate N-succinimidyl ester (CFSE) was from Sigma. Quantitative PCR (qPCR) SYBR mix was from TIANGEN.

Constructs, RNA Interference and Lentiviral Vectors Production. For knockdown experiments, the lentiviral vector FG12 described previously by Qin ${ }^{17}$ was used. Short hairpin shRNAs in the pLKO.1 lentiviral vector was purchased from Open Biosystem (Thermo Fisher Scientific, Pittsburgh PA, USA). The RNAi sequences specifically targeting E2-2 mRNA were as follows: E2-2i 1\#, 5'-GAAAGGAATCTGAATCCGAAA-3'; E2-2i 2\#, 5'-CACGAAATCTTCGGAGGACAA-3'. The U6 RNAi sequence cassette was then subcloned into FG12. An RNAi construct with only U6 promoter was used as control. Vectors were harvested at $48 \mathrm{~h}$ post transfection of $293 \mathrm{~T}$ cells with lentiviral vector, Gag-Pol $(\Delta \mathrm{NRF})$ and Env (Vsvg). GEN2.2 cells were transduced with lentiviral vectors for $3 \mathrm{~h}$ and polybrene $(4 \mu \mathrm{g} / \mathrm{ml})$ added.

RT- PCR and ELISA. For E2-2 knockdown, GEN2.2 cells were transduced by lentiviral vectors with or without shRNA for E2-2, and $\mathrm{GFP}^{+}$cells were sorted at 3-5 days post infection. Cells were resuspended in Trizol reagent (Invitrogen), total RNA was isolated according to Molecular Cloning: A Laboratory Manual and reverse transcribed using a poly-dT oligonucleotide (Invitrogen) and M-MLV Reverse Transcriptase (Promega) at $42^{\circ} \mathrm{C}$ for $1 \mathrm{hr}$. PCR assays were carried out with Corbett 6200/6600 qPCR System using $5 \mu \mathrm{l} \mathrm{cDNA}$ template, $0.25 \mu \mathrm{M}$ of each primer and Taq polymerase in $1 \times$ SYBR Green Mix. Reaction conditions were as follows: a $10 \mathrm{~min}$ pre-denaturation step at $95^{\circ} \mathrm{C}$ was followed by 40 cycles of $10 \mathrm{~s}$ at $95^{\circ} \mathrm{C}, 15 \mathrm{~s}$ at $55^{\circ} \mathrm{C}$, and $15-30 \mathrm{~s}$ at $72^{\circ} \mathrm{C}$. Primers used for the measurement of indicated gene expression were as follows: E2-2, 5'-GAGTGTCTCCTCTGGCAGC-3' and 5'-CCATGTGATTCGATGCGTC-3'; CLEC4C (BDCA2), 5'-ACTGGGATGCAATCTTGGAC-3' and 5'-GATCTGACAGCCCCAGAAAA-3'; Siglec-6, 5' - AAGGGGCTGATGTTCCAGTG-3' and 5'- ATGCAGCATTGTCCCTCCTC-3'; TCL1B, 5'-TTCCAGTTTCTGGGAAATAGCAG-3' and 5'-TCTCCGGCTGATATGTTAGGAC-3'; GZMB, 5'-TACCATTGAGTTGTGCGTGGG-3' and 5'-GCCATTGTTTCGTCCATAGGAGA-3'. Quantitation was normalized to an endogenous EF1 $\alpha$ control. For an ELISA, infected GEN2.2 cells were cultured in the presence of different concentrations of type B CpG (ODN2006, Invitrogen) for $20 \mathrm{hr}$. Culture supernatants were collected and analyzed by human-specific IFN $\alpha$ and IL-6 ELISA kit (Bender MedSystems).

Western Blot Analysis. Cells were lysed at $48 \mathrm{~h}$ after transfection of E2-2 expression plasmid and control shRNA or E2-2-specific shRNA using PEI (Sigma). Samples were separated by SDS-PAGE and transferred to PVDF membrane. After blocking in TBS containing 5\% skim-milk, the blots were probed with indicated antibodies.

Flow Cytometry. Monoclonal antibodies to CD3, CD11c, CD14, CD16, CD20, CD123, and HLA-DR conjugated to FITC, APC-Cy7, APC, PerCP_Cy5.5 were purchased from BioLegend, and Live/Dead Fixable Yellow Dead Cell Stain Kit from Invitrogen. Mouse monoclonal antibody to human Siglec-6 was prepared in mouse according to Current Protocols in Immunology. Single cell suspensions were stained and analyzed with FACS Fortessa (BD). Cell sorting was performed with FACS Aria III sorter (BD) and data were analyzed with Summit software (Dako Cytomation). 
A

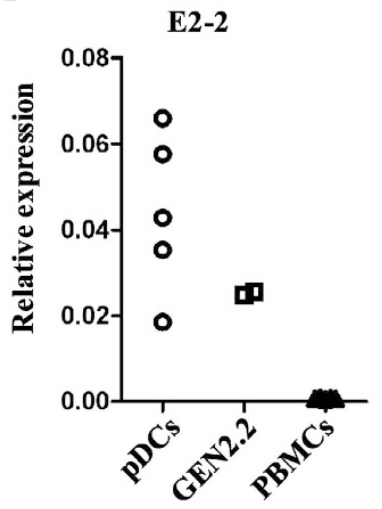

B

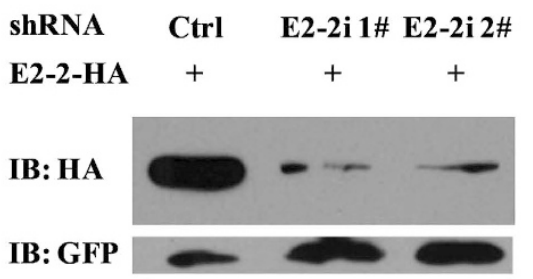

C

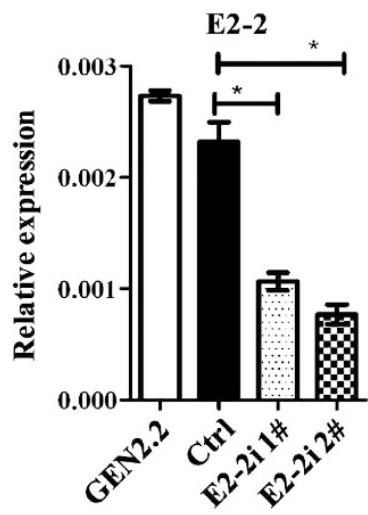

Figure 1. E2-2 expression in GEN2.2 is silenced by shRNAs. (A) E2-2 expression in GEN2.2 cells, purified human pDCs and PBMCs $(\mathrm{n}=5)$ was measured by real-time PCR. (B) Immunobloting analysis of E2-2 in lysates of 293T cells transfected with E2-2 overexpression plasmid and control shRNA or E2-2-specific shRNA. GFP was used as a loading control. (C) E2-2 expression in GEN2.2 cells transduced with or without control shRNA or E2-2-specific shRNA was measured by real-time PCR.. ${ }^{*}$ indicated $\mathrm{p}<0.05$. All the gels were run under the same experimental conditions as detailed in the Methods section, and full-length blots were cropped for final display.

Isolation of Human Primary pDCs. Human PBMCs were stained with mouse antibody mix to human CD3, CD14, CD16, and CD19. Lineage-positive cells were depleted by goat anti-mouse IgG $(\mathrm{H}+\mathrm{L})$ microbeads with MACS Columns and MACS Separators (Miltenyi Biotec). The negative cells were stained with CD123 and HLA-DR. CD123 ${ }^{+} \mathrm{HLA}_{-} \mathrm{DR}^{+}$cells were sorted by FACS (>95\% purity).

T cells Proliferation. GEN2.2 cells transduced with lentiviral vector expressing shRNAs of E2-2 at 3-5 days post infection were sorted by FACS Aria III sorter (BD). Naive CD4 ${ }^{+}$CD45RA ${ }^{+}$T lymphocytes ( $>95 \%$ purity) were isolated from human PBMC via negative immunomagnetic depletion (Miltenyi Biotech), labeled with $5 \mu \mathrm{M}$ CFSE for $10 \mathrm{~min}$ at room temperature and quenched by adding an equal volume of FBS. GEN2.2 and T cell mix was cultured in a 96-well round-bottom plate. 7 days later, cells were harvested and analyzed by flow cytometry.

Gene Expression Profiling. For microarray analysis, E2-2 shRNA transduced GEN2.2 cells were sorted and resuspended in Trizol reagent (Invitrogen), and total RNA was reverse transcribed, amplified with a MessageAmp ${ }^{\mathrm{TM}}$ Premier RNA Amplification Kit (Ambion) and labeled, fragmented and hybridized to Human Genome U133 Plus 2.0 arrays (Affymetrix). Chips were analyzed using a Laser Scan Confocol microarray reader (CapitalBio, Beijing, China). Raw data were transformed with the RMA algorithm, which yields a normalized expression value, and "absent" and "present" calls. Normal gene expression profiles of human blood leukocytes were derived from supplementary materials and EBI Array Express data set E-TABM-34.

Statistical Analysis. Statistical significance was determined by unpaired, two-tailed Student's t test. $P$ value $<0.05$ was considered statistically significant. Data were analyzed with GraphPad Prism software version 5.0 (GraphPad Software). The methods used for analysis of microarray data were described below.

\section{Results}

Short hairpin RNAs knock down E2-2 in GEN2.2 cells. The GEN2.2 cells resemble primary human pDCs with similar phenotype and function ${ }^{18,19}$. E2-2 was expressed abundantly in human pDCs, and at similar levels in GEN2.2 cells (Fig. 1A). We selected four short hairpin RNAs (shRNAs) for E2-2, all of which could knock down E2-2 expression in transfected 293T cells (Figure S1). Of the four shRNAs, two could knockdown E2-2 expression efficiently (Fig. 1B and Figure S2). These two shRNAs were chosen for construction of lentiviral vectors for E2-2 knockdown in GEN2.2 cells. The lentiviral vector carries independent GFP expression cassette, which is a reporter and selection marker for cells successfully transduced by E2-2 shRNA. GEN2.2 cells can hardly be transfected with plasmid and lentiviral vector enables efficient gene transfer in the absence of cell phenotypic and functional maturation ${ }^{20}$.

Quantitative RT-PCR (qRT-PCR) with $\mathrm{GFP}^{+}$GEN2.2 cells showed that more than $50 \%$ of E2-2 transcription was significantly suppressed (Fig. 1C). Comparable E2-2 expression was observed in GEN2.2 cells transduced with control lentiviral vector, which indicated that infection didn't affect E2-2 expression. 
E2-2 silence in GEN2.2 cells abrogates IFN- $\alpha$ production but induces proliferation of naïve T lymphocytes.. GEN2.2 cells produce IFN- $\alpha$ in response to unmethylated DNA (CpG). IFN- $\alpha$ secretion after CpG B $(0 \sim 1 \mu \mathrm{M})$ stimulation was detected in control GEN2.2 cells and peaked at $0.2 \mu \mathrm{M}$. However, E2-2 knockdown GEN2.2 cells could barely secret any IFN- $\alpha$ in response to CpG B (Fig. 2A). Additionally, the level of inflammatory cytokine IL-6 was reduced to $30 \%$ of control cells (Fig. 2B). Thus, E2-2 was indispensable for $\mathrm{pDCs}$ to yield tremendous IFN- $\alpha$ upon stimulation.

After 48h activation with IL-3 plus CD40L, with virus or with the three signals, matured GEN2.2 cells can stimulate naive T lymphocyte proliferation ${ }^{18}$. Without activation, GEN2.2 cells show poor capacity to prime T cells. We co-cultured the GEN2.2 cells with freshly isolated naïve $\mathrm{CD} 4^{+} \mathrm{T}$ cells, which were pre-labeled with CFSE. GEN2.2 cells and control lentiviral vector transduced cells could barely induce $\mathrm{T}$ cell proliferation $(\sim 3 \%)$. However, without any stimulation, $\sim 50 \% \mathrm{~T}$ cells proliferated once co-cultured with E2-2 knockdown GEN2.2 cells (Fig. 2C). FACS analysis of costimulatory molecules showed increased expression of CD40, CD83 and CD86 after E2-2 down-regulation (Figure S3). Thus, we concluded that GEN2.2 cells acquired antigen presentation capacity after E2-2 knockdown.

E2-2 reduction in GEN2.2 cells down-regulates $\mathrm{PDC}$ signature genes and up-regulates CDC signature genes. E2-2 controls the gene expression program of mouse pDCs. As a transcription factor, E2-2 can directly bind the regulatory regions of some $\mathrm{pDC}$ specific genes ${ }^{14}$. To identify gene program regulated by E2-2 in human pDCs, E2-2 knockdown GEN2.2 cells were analyzed by expression microarrays. E2-2 expression was downregulated to about $50 \%$ as compared with control cells by all eight probesets. Expression of housekeeping genes (GAPDH and Actin) were at the same level in E2-2 knockdown and control GEN2.2 cells (data not shown). Significance analysis of microarray (SAM) showed that 827 probe sets were differentially expressed in E2-2 knockdown and control GEN2.2 cells by more than two-folds: 628 probe sets were expressed at higher abundance in E2-2 knockdown GEN2.2 cells as compared with control GEN2.2 cells. And 199 probe sets were expressed at lower abundance in E2-2 knockdown GEN2.2 cells as compared with control GEN2.2 cells (Fig. 3A).

To exclude those leukemia-related genes ${ }^{21}$ and observe the change of DC specific gene expression program, we constructed a list of "DC signature" probe sets which were defined by highest expression in $\mathrm{pDCs}$ or $\mathrm{cDCs}$ via evaluation of overall gene expression in human blood leukocytes with Wilcoxon signed-rank test $(\mathrm{P}<0.05)$. Gene expression profiles of monocytes, neutrophils, $\mathrm{pDCs}, \mathrm{BDCA1}{ }^{+} \mathrm{cDCs}$, $\mathrm{BDCA}^{+}{ }^{+} \mathrm{CDCs}, \mathrm{CD} 8^{+} \mathrm{T}, \mathrm{CD} 4^{+} \mathrm{T}, \mathrm{NK}$ and $\mathrm{B}$ cells were retrieved from public databases of previous publication ${ }^{1}$.

Then the overlap of these "DC signature" genes and differentially expressed genes in E2-2 knockdown GEN2.2 cells showed that all $\mathrm{cDC}$ signature sets (17/17) were up-regulated while most pDC signature sets (15/18) were down-regulated (Table 1). Human pDC surface markers like CLEC4C (BDCA2)(Fig. 3B), LILRA4 (ILT7) were included in the down-regulated set, while cDC highly expressed class II transactivator (CIITA), which is essential for MHCII expression, was present in the up-regulated set (Fig. 3A). In accordance with that, expression levels of HLA-DQA1, HLA-DRB1, HLA-DQB1 and HLA-DPB1 were increased (Fig. 3A and Table 1).

Among the $\mathrm{pDC}$ signature genes, GZMB was the most down-regulated gene $(\mathrm{E} 2-2 \mathrm{i} / \mathrm{Ctrl}$ ratio $=0.11$, Fig. 3B). Granzymes such as GZMB represent a major component of the granules of cytotoxic cells, like cytotoxic T lymphocytes (CTLs) and NK cells. CTLs recognize target cells and secrets granules, which will induce cell apoptosis. Recent report suggested that human pDCs were also an abundant source of enzymatically active GZMB protein and they may produce GZMB in amounts that exceed GZMB produced by classical cytotoxic lymphocytes ${ }^{22}$. These $\mathrm{pDC}$-derived GZMB suppresses $\mathrm{T}$ cell proliferation in perforin-independent manner. Down-regulated GZMB in E2-2 knockdown GEN2.2 cells may result in higher proliferation of T cells. For mouse, GZMB could only be detected in NK cells and mast cells but not pDCs.

Human specific genes regulated by E2-2. To define species-specific features of human pDCs regulated by E2-2, we submitted above differentially expressed genes to the Database for Annotation, Visualization and Integrated Discovery (DAVID) and analyzed them with functional annotation tool ${ }^{23}$. A gene list encompassing thirty genes which were expressed in human but not mouse was produced via limiting annotations by homo sapiens or mus musculus and aligning the two groups (Table 2). We found some well characterized genes (such as CLEC4C and LILRA4) as well as several novel genes not reported previously.

Toll-like receptors play an essential role in innate immunity against pathogens. pDCs recognize DNA virus and CpG or single-stranded viral RNA for their selectively expression of TLR7 and TLR9. Associated with MyD88, TLR10 is weakly expressed in human $\mathrm{pDCs}^{8}$ and preferentially expressed on $\mathrm{CD}^{2} \mathrm{a}^{+}$subset of $\mathrm{CDCs}^{24}$. Expression of TLR10 has not been detected in mouse ${ }^{25}$. Human TLR10 is an anti-inflammatory pattern-recogenition receptor without known ligand ${ }^{24}$. Once E2-2 was down-regulated, GEN2.2 promoted $\mathrm{CDC}$ signature genes expression and TLR10 would be one of them.

TCL1B is a protooncogene and shares $60 \%$ sequence homology with the other family member TCL1A. Intracellular TCL1A expression is considered to be a marker for plasmacytoid dendritic cell leukemia. About $37 \%$ blood primary pDCs express TCL1A, however, it's highly expressed in most pDC leukemia 
A

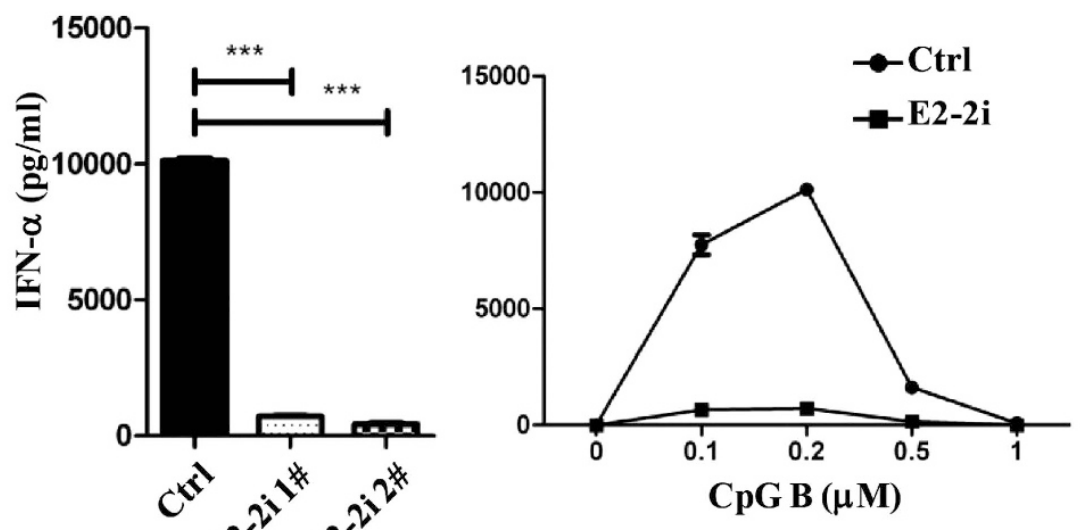

B
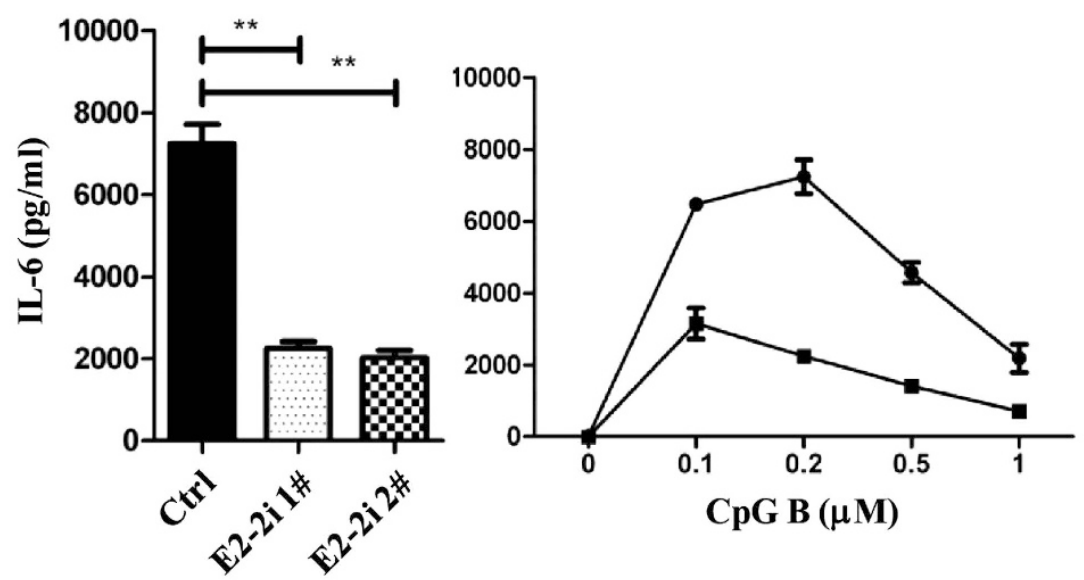

C $\mathbf{T}$

T+GEN2.2
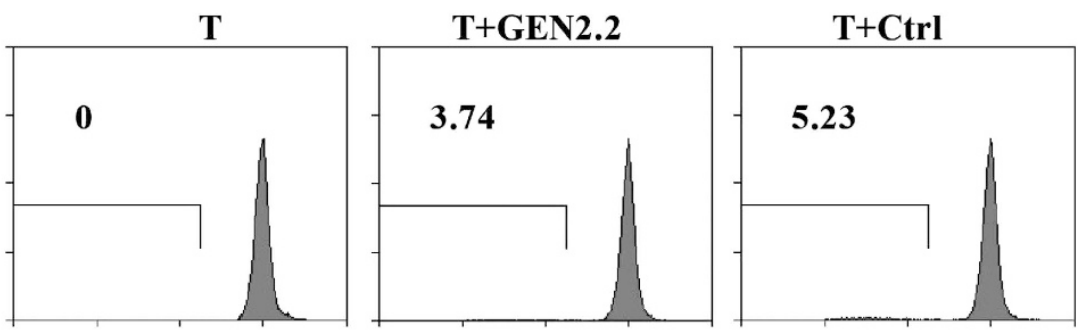

T+E2-2i 1\#

T+E2-2i 2\#
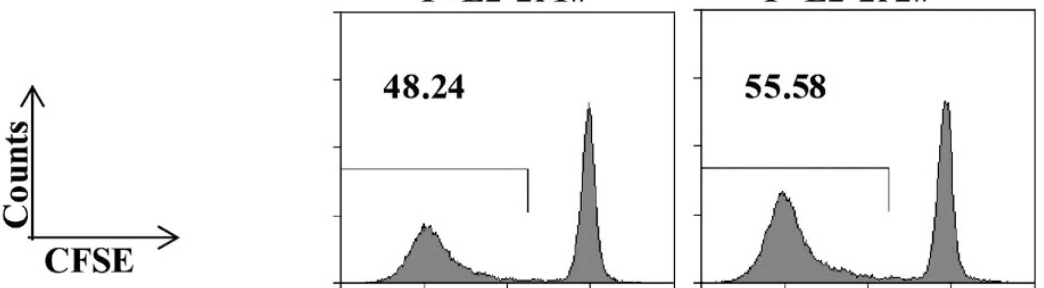

Figure 2. Decreased IFN $\alpha$ and IL-6 production and higher T cell proliferation by E2-2 knockdown GEN2.2 cells. (A) and (B) GEN2.2 cells were incubated with CpG B for $20 \mathrm{~h}$. Different concentrations $(0 \sim 1 \mu \mathrm{M})$ of CpG B stimulated cell supernantant was analyzed (right panel) and $0.2 \mu \mathrm{M}$ was shown (left panel). (A) IFN- $\alpha$ in supernatants of E2-2 knockdown and control GEN2.2 cells was measured by ELISA. *** indicated $p<0.001$. (B) IL-6 in supernatants of E2-2 knockdown and control GEN2.2 cells was measured by ELISA. ${ }^{* *}$ indicated $p<0.01$. (C) T cell priming capacity of E2-2 knockdown GEN2.2 cells. GEN2.2 cells transduced with or without control shRNA or E2-2-specific shRNA were co-cultured with CD4 ${ }^{+} \mathrm{CD} 45 \mathrm{RA}^{+}$ $\mathrm{T}$ cells, which were purified and labeled with CFSE. 7 days later, cells were analyzed for CFSE levels by flow cytometry. Numbers indicated percentage of $\mathrm{CFSE}^{\text {low }}$ (proliferated) cells. Data are representative of at least three independent experiments. 
A

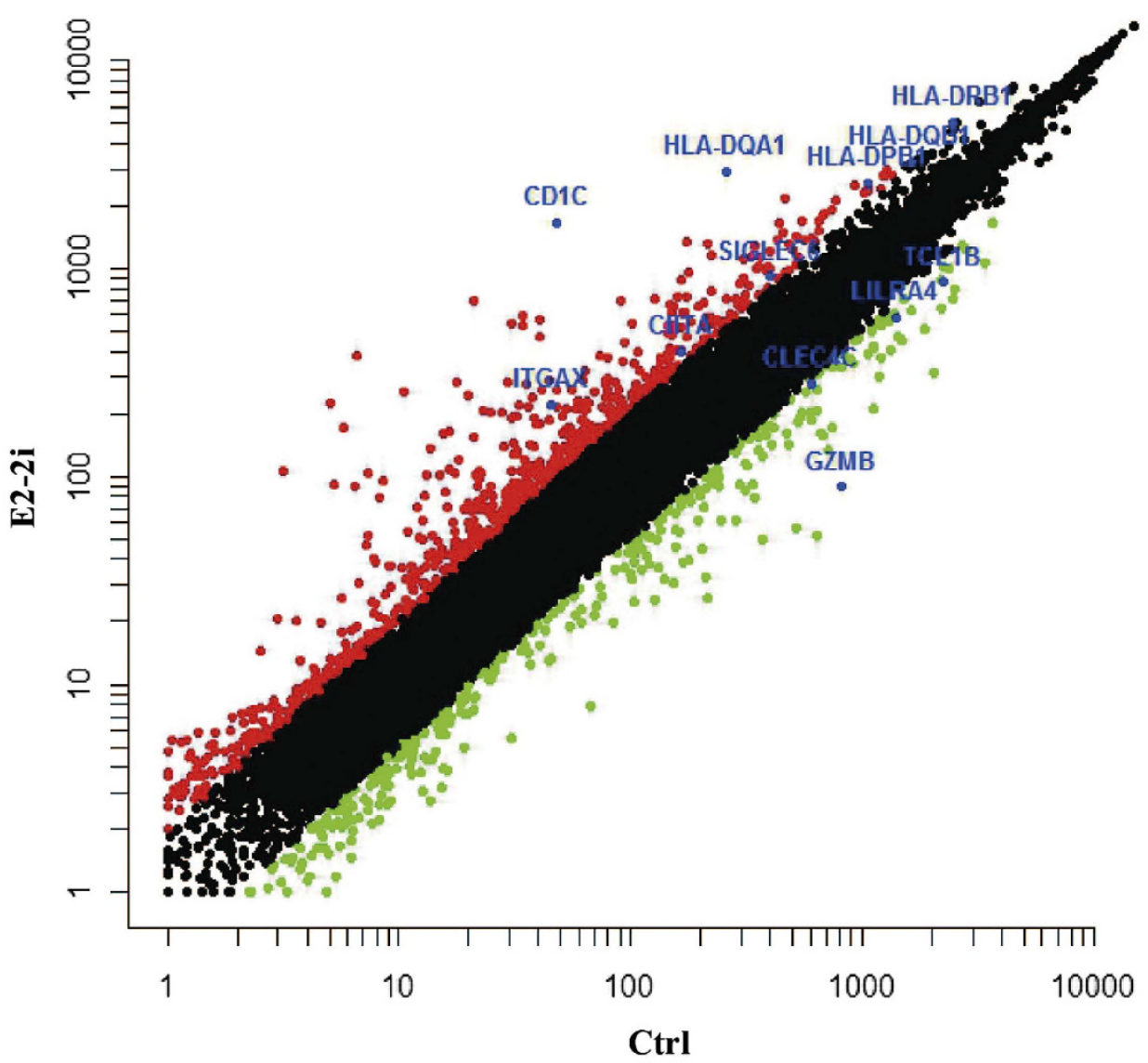

B

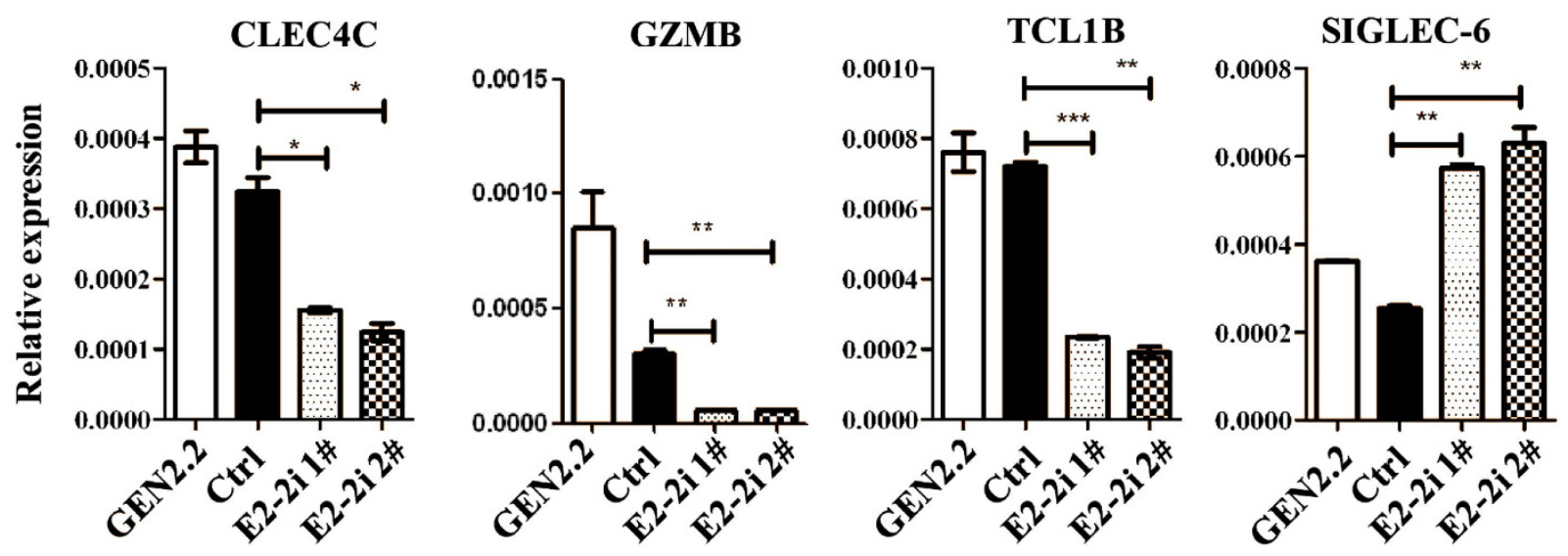

Figure 3. Gene program regulated by E2-2 in human pDCs. (A) Pairwise comparison of expression profile for E2-2 knockdown and control GEN2.2 cells. The scatter plot represents normalized log intensities of individual probes, with the probes increased or decreased $>2$ fold in GEN2.2 cells with E2-2-specific shRNA (E2-2i) and control shRNA (Ctrl) indicated in red and green, respectively. Some probes are highlighted in blue. (B) Expression of CLEC4C, GZMB, TCL1B and Siglec-6 in GEN2.2 cells with or without control shRNA or E2-2-specific shRNA. ${ }^{*},{ }^{* *}$ and ${ }^{* *}$ indicated $\mathrm{p}<0.05, \mathrm{p}<0.01$ and $\mathrm{p}<0.001$.

samples $^{26}$. Intracellular staining showed that all GEN2.2 cells express TCL1A (Figure S4). Our results showed that TCL1B was down-regulated after E2-2 silence (Fig. 3B).

Sialic acid-binding immunoglobulin-like lectin 6 (Siglec-6) is mainly expressed on immune cells and placental trophoblast ${ }^{27,28}$. As an inhibitory receptor, Siglec-6 has a dominant effect on both the proliferation and effector functions of tissue-like memory B cells ${ }^{29}$. It has been reported that Siglec- 6 is expressed 


\begin{tabular}{|c|c|c|}
\hline Gene symbol & Gene title & Ratio \\
\hline \multicolumn{3}{|c|}{ cDC signature genes } \\
\hline LY86 & lymphocyte antigen 86 & 7.6826 \\
\hline HLA-DQA1 & major histocompatibility complex, class II, DQ alpha 1 & 4.7388 \\
\hline EMP1 & epithelial membrane protein 1 & 3.6011 \\
\hline HLA-DQB1 & major histocompatibility complex, class II, DQ beta 1 & 2.6273 \\
\hline PSEN2 & presenilin 2 (Alzheimer disease 4 ) & 2.533 \\
\hline HLA-DPB1 & major histocompatibility complex, class II, DP beta 1 & 2.4325 \\
\hline DISC1 & disrupted in schizophrenia 1 & 2.4288 \\
\hline CIITA & class II, major histocompatibility complex, transactivator & 2.4064 \\
\hline CSRP1 & cysteine and glycine-rich protein 1 & 2.2192 \\
\hline PPM1H & protein phosphatase $1 \mathrm{H}$ (PP2C domain containing) & 2.2087 \\
\hline KCNMB1 & $\begin{array}{l}\text { potassium large conductance calcium-activated channel, subfamily } \mathrm{M} \text {, } \\
\text { beta member } 1\end{array}$ & 2.2002 \\
\hline ENTPD1 & ectonucleoside triphosphate diphosphohydrolase 1 & 2.1907 \\
\hline CENTA2 & ArfGAP with dual PH domains 2 & 2.1818 \\
\hline IER5 & immediate early response 5 & 2.1753 \\
\hline NAB2 & NGFI-A binding protein 2 (EGR1 binding protein 2 ) & 2.1598 \\
\hline SPRY2 & sprouty homolog 2 (Drosophila) & 2.0886 \\
\hline ATP1B1 & ATPase, $\mathrm{Na}+/ \mathrm{K}+$ transporting, beta 1 polypeptide & 2.0514 \\
\hline \multicolumn{3}{|c|}{ pDC signature genes } \\
\hline GZMB & granzyme B & 0.1093 \\
\hline RGS7 & regulator of G-protein signaling 7 & 0.2939 \\
\hline COBLL1 & COBL-like 1 & 0.3393 \\
\hline SRPX & sushi-repeat-containing protein, $\mathrm{X}$-linked & 0.3716 \\
\hline TCL1B & T-cell leukemia/lymphoma 6 & 0.3829 \\
\hline LILRA4 & $\begin{array}{l}\text { leukocyte immunoglobulin-like receptor, subfamily A (with TM } \\
\text { domain), member } 4\end{array}$ & 0.417 \\
\hline MAP1A & microtubule-associated protein $1 \mathrm{~A}$ & 0.4371 \\
\hline DUSP5 & dual specificity phosphatase 5 & 0.4389 \\
\hline MYB & v-myb myeloblastosis viral oncogene homolog (avian) & 0.4568 \\
\hline CLEC4C & C-type lectin domain family 4 , member C & 0.4609 \\
\hline MLF1IP & MLF1 interacting protein & 0.461 \\
\hline SLC12A3 & solute carrier family 12 , member 3 & 0.4707 \\
\hline RRBP1 & transforming growth factor, beta-induced, $68 \mathrm{kDa}$ & 0.4711 \\
\hline TGFBI & ribosome binding protein 1 homolog $180 \mathrm{kDa}(\mathrm{dog})$ & 0.4711 \\
\hline KCNK1 & potassium channel, subfamily $\mathrm{K}$, member 1 & 0.4809 \\
\hline ENPP2 & ectonucleotide pyrophosphatase/phosphodiesterase 2 & 2.0998 \\
\hline RASGEF1B & RasGEF domain family, member $1 \mathrm{~B}$ & 2.4254 \\
\hline DAB2 & disabled homolog 2, mitogen-responsive phosphoprotein (Drosophila) & 3.4648 \\
\hline
\end{tabular}

Table 1. Differentially expressed DC signature genes in E2-2 knockdown GEN2.2 cells.

on plasmacytoid dendritic cell leukemia but not cutaneous myeolomonocytic leukemia (c-AML) ${ }^{30}$. qRT-PCR result confirmed augmented expression of Siglec-6 in GEN2.2 cells after E2-2 knockdown (Fig. 3B). To test Siglec-6 expression on human primary pDCs, we prepared monoclonal antibody against human Siglec-6, and the antibody was conjugated with PE (CD327-PE). According to Siglec-6 expression, human pDCs in PBMCs were divided into two subsets: Siglec-6 ${ }^{\text {high }}$ pDCs and Siglec- $6^{\text {low }}$ pDCs (Fig. 4A). Different Siglec-6 expression at mRNA level was validated by qRT-PCR. Siglec-6 ${ }^{\text {low }}$ pDCs express $\sim 4$ folds higher E2-2 than Siglec- $6^{\text {high }}$ pDCs (Fig. $4 \mathrm{~B}$ ). Besides, there were three E2-2 binding motifs (CANNTG) 500 bp upstream ATG of Siglec- 6 coding region, which were potential binding sites for E2-2 (data not shown). Thus, we propose that E2-2 inhibits Siglec-6 expression in pDCs.

Previously a ChIP-on-chip array was performed in human pDC cell line CAL-1. A high-confidence list of 2,477 genes was defined as E2-2 binding targets ${ }^{15}$. The overlap of these targets and the differentially 


\begin{tabular}{|c|c|c|}
\hline Gene Symbol & Gene Title & Ratio \\
\hline HLA-DQA1 & $\begin{array}{l}\text { major histocompatibility complex, } \\
\text { class II, DQ alpha } 1\end{array}$ & 4.7388 \\
\hline HLA-DRB1 & $\begin{array}{l}\text { major histocompatibility complex, } \\
\text { class II, DR beta } 1\end{array}$ & 3.6552 \\
\hline C11orf21 & $\begin{array}{c}\text { chromosome } 11 \text { open reading } \\
\text { frame } 21\end{array}$ & 3.6179 \\
\hline $\mathrm{CA} 2$ & carbonic anhydrase II & 3.1831 \\
\hline ANKH & $\begin{array}{l}\text { ankylosis, progressive homolog } \\
\text { (mouse) }\end{array}$ & 3.0989 \\
\hline HLA-DQB1 & $\begin{array}{l}\text { major histocompatibility complex, } \\
\text { class II, DQ beta } 1\end{array}$ & 2.7546 \\
\hline LOC100288152 & hypothetical protein LOC100288152 & 2.7061 \\
\hline TLR10 & toll-like receptor 10 & 2.687 \\
\hline HIST1H2BD & histone cluster $1, \mathrm{H} 2 \mathrm{bd}$ & 2.5222 \\
\hline HIST1H2BC & histone cluster $1, \mathrm{H} 2 \mathrm{bc}$ & 2.4774 \\
\hline HLA-DPB1 & $\begin{array}{l}\text { major histocompatibility complex, } \\
\text { class II, DP beta } 1\end{array}$ & 2.4325 \\
\hline Siglec-6 & sialic acid binding Ig-like lectin 6 & 2.3482 \\
\hline $\mathrm{H} 2 \mathrm{BFS}$ & H2B histone family, member $\mathrm{S}$ & 2.2932 \\
\hline ZNF827 & zinc finger protein 827 & 2.2415 \\
\hline CLEC2B & $\begin{array}{l}\text { C-type lectin domain family } 2 \text {, } \\
\text { member B }\end{array}$ & 2.2331 \\
\hline SP140L & SP140 nuclear body protein-like & 2.1677 \\
\hline C1orf228 & $\begin{array}{c}\text { chromosome } 1 \text { open reading frame } \\
228\end{array}$ & 2.0496 \\
\hline SPTBN1 & spectrin, beta, non-erythrocytic 1 & 0.4835 \\
\hline SERHL2 & serine hydrolase-like 2 & 0.4626 \\
\hline CLEC4C & $\begin{array}{l}\text { C-type lectin domain family } 4 \text {, } \\
\text { member C }\end{array}$ & 0.4609 \\
\hline LOC150568 & hypothetical LOC150568 & 0.4609 \\
\hline MAP1A & microtubule-associated protein $1 \mathrm{~A}$ & 0.4371 \\
\hline LILRA4 & $\begin{array}{l}\text { "leukocyte immunoglobulin-like } \\
\text { receptor, subfamily A (with TM } \\
\text { domain), member 4" }\end{array}$ & 0.417 \\
\hline FLJ35024 & hypothetical LOC401491 & 0.4129 \\
\hline IGHA1 & $\begin{array}{c}\text { immunoglobulin heavy constant } \\
\text { alpha } 1\end{array}$ & 0.4067 \\
\hline IGHM & immunoglobulin heavy constant $\mathrm{mu}$ & 0.3864 \\
\hline TCL1B & T-cell leukemia/lymphoma $1 \mathrm{~B}$ & 0.3829 \\
\hline IGLC1 & $\begin{array}{l}\text { Immunoglobulin lambda constant } 1 \\
\text { (Mcg marker) }\end{array}$ & 0.3147 \\
\hline ZNF385B & zinc finger protein $385 \mathrm{~B}$ & 0.234 \\
\hline MAP1B & microtubule-associated protein $1 \mathrm{~B}$ & 0.1995 \\
\hline
\end{tabular}

Table 2. Differentially expressed human specific genes in E2-2 knockdown GEN2.2 cells.

expressed genes comprised 90 genes, which were bound to and regulated directly by E2-2 (Table S1). Other than well known pDC and cDC specific genes, we identified genes that were not yet known to be involved in the biology of pDCs. Take SPTDN1 for example, recent report showed that it was an important host target for IL-27 to inhibit HIV-1 infection in human macrophages ${ }^{31}$. pDCs could be infected by HIV and whether SPTBN1 was included was still unknown. Among these genes bound to and regulated directly by E2-2, C20orf26, CD1E, FCRL4, LILRA4, TCL1B and TP53I3 were human specific genes. FCRL4 was one of the most up-regulated genes $(\mathrm{E} 2-2 \mathrm{i} / \mathrm{Ctrl}$ ratio $=17.77)$ in E2-2 knockdown GEN2.2 cells. As an inhibitory receptor, FCRL4 contributes to HIV-associated human B cell exhaustion ${ }^{29}$, and is considered as a molecular switch in B cells to dampen adaptive immune signaling and enhance innate signaling in response to chronic antigenic stimulation ${ }^{32}$. pDCs has been described as the bridge for innate and adaptive immunity ${ }^{33}$. Whether the function of FCRL4 on pDCs as a molecular switch is 
A

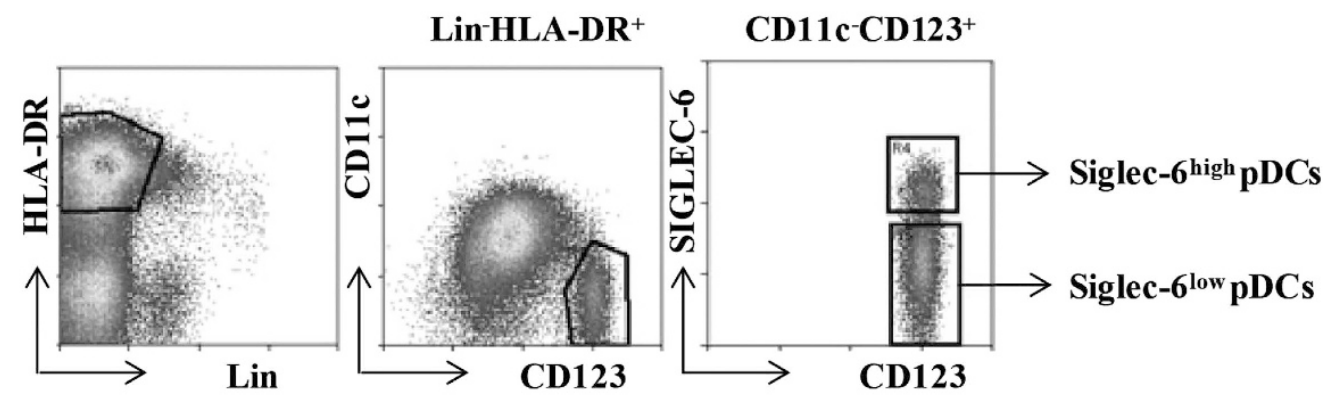

B

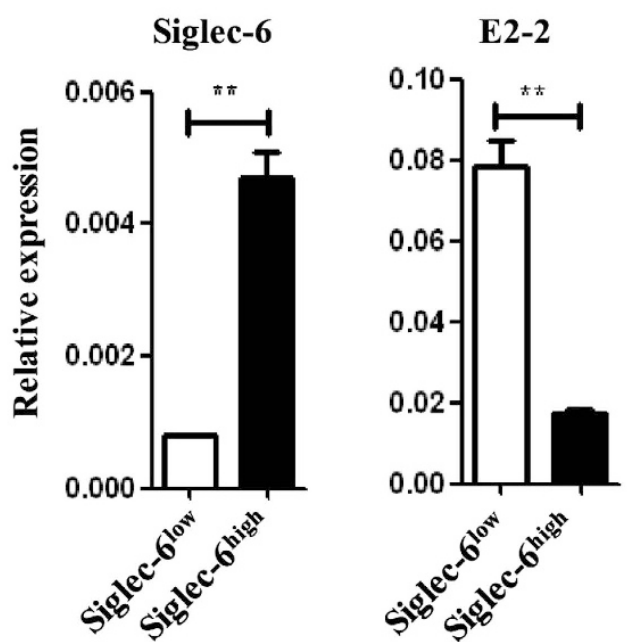

Figure 4. Siglec-6 and E2-2 expression on human pDC subsets. (A) Flow cytometry analysis of Siglec-6 expression on human pDCs from lineage negative PBMCs. Primary pDCs were marked as HLA-DR-CD11c $\mathrm{CD}_{12} 3^{+}$and divided into two subsets according to Siglec-6 expression. (B) Siglec-6 and E2-2 expression in Siglec- $6^{\text {high }}$ or Siglec- $6^{\text {low }}$ pDC subsets. ${ }^{* *}$ indicated $\mathrm{p}<0.01$. Data are representative of at least three donors.

inversed has never been reported. Thus, we have defined novel specific genes regulated by E2-2 which can be candidates for further investigation in human pDCs.

\section{Discussion}

Here we investigated the function of E2-2 in human pDC functions. Consistent with that in mouse, loss of E2-2 in human pDCs eliminated IFN response to unmethylated DNA, inhibited pDC signature genes expression and elevated antigen presentation ability without any stimulation.

Heterogeneity of pDCs has been proposed ever since 2005. Kamogawa-Schifter provides the first evidence that murine pDCs can be divided into two subsets according to the expression of Ly49 $\mathrm{Q}^{34}$. Ly49 $\mathrm{Q}^{-}$pDCs are procursors of Ly49Q ${ }^{+}$pDCs. RAG1, Ig rearrangement products ${ }^{35}$ and CD $4^{36}$ are also used to demonstrate that murine pDCs constitute a heterogeneous cell population. CCR9 has been used to distinguish functionally distinct $\mathrm{pDC}$ subsets but finally it proves that $\mathrm{B} 220^{+} \mathrm{CCR} 9^{-} \mathrm{DCs}$ are not $\mathrm{pDCs}$ but precursors of $\mathrm{cDCs}^{37,38}$. For human pDCs, CD2 contributes to define pDC heterogeneity ${ }^{39}$. Here we identify two pDC subsets: Siglec- $6^{\text {high }}$ and Siglec- $6^{\text {low }}$. Both of them were Lin-HLA-DR+CD11c-CD123+ and express the transcription factor E2-2. Siglec- $6^{\text {high }}$ pDCs express lower E2-2 and their developmental pathway should be well studied to ascertain whether they are pDC precursor or cDC precursor or another cell subset.

Most Siglecs have two conserved intracellular domains: ITIM and ITIM-like motif, capable of inhibiting signaling. For example, mouse eosinophils and activated T cells express Siglec-F, which is a functional counterpart of human Siglec-8 and controls responses of eosinophils and helper T cells ${ }^{40,41}$. Loss of Siglec-F will increase bone-marrow, blood and tissue eosinophilia in a model of induced lung allergy ${ }^{42}$. In HIV-associated B cell exhaustion, Siglec-6 is reported to inhibit BCR-induced proliferation and effector function of tissue-like memory B cells ${ }^{29}$. The role of Siglec- 6 in human pDCs has never been investigated. Recent report show extensive and direct TLR-Siglec interactions negatively regulate the function of multiple TLRs. Among them, Siglec- 6 weakly interacted with TLR9 and strongly interacted with TLR10 expressed from human THP-1 ${ }^{43}$. After E2-2 knockdown, Siglec-6 and TLR10 were up-regulated and their strong interaction may inhibit pDC response to TLR ligand (CpG B) and explain abrogated IFN- $\alpha$ production. 
Importantly, our study confirms the function of E2-2 in human pDCs and reveals a number of human genes regulated by E2-2, delineating a non-redundant role of E2-2 in refining species gap between human and mouse pDCs.

\section{References}

1. Robbins, S. H. et al. Novel insights into the relationships between dendritic cell subsets in human and mouse revealed by genome-wide expression profiling. Genome Biol. 9, R17 (2008).

2. Crozat, K. et al. Comparative genomics as a tool to reveal functional equivalences between human and mouse dendritic cell subsets. Immunol. Rev. 234, 177-198 (2010).

3. Asselin-Paturel, C. et al. Mouse type I IFN-producing cells are immature APCs with plasmacytoid morphology. Nat. Immunol. 2, 1144-1150 (2001)

4. Bjorck, P. Isolation and characterization of plasmacytoid dendritic cells from Flt3 ligand and granulocyte-macrophage colonystimulating factor-treated mice. Blood 98, 3520-3526 (2001).

5. Ito, T., Kanzler, H., Duramad, O., Cao, W. \& Liu, Y. J. Specialization, kinetics, and repertoire of type 1 interferon responses by human plasmacytoid predendritic cells. Blood 107, 2423-2431 (2006).

6. Edwards, A. D. et al. Toll-like receptor expression in murine DC subsets: lack of TLR7 expression by CD8 alpha ${ }^{+}$DC correlates with unresponsiveness to imidazoquinolines. Eur. J. Immunol. 33, 827-833 (2003).

7. Krug, A. et al. Toll-like receptor expression reveals CpG DNA as a unique microbial stimulus for plasmacytoid dendritic cells which synergizes with CD40 ligand to induce high amounts of IL-12. Eur. J. Immunol. 31, 3026-3037 (2001).

8. Hornung, V. et al. Quantitative expression of toll-like receptor 1-10 mRNA in cellular subsets of human peripheral blood mononuclear cells and sensitivity to CpG oligodeoxynucleotides. J. Immunol. 168, 4531-4537 (2002).

9. Dzionek, A. et al. BDCA-2, a novel plasmacytoid dendritic cell-specific type II C-type lectin, mediates antigen capture and is a potent inhibitor of interferon alpha/beta induction. J. Exp. Med. 194, 1823-1834 (2001).

10. Rahim, M. M. et al. Ly49Q positively regulates type I IFN production by plasmacytoid dendritic cells in an immunoreceptor tyrosine-based inhibitory motif-dependent manner. J. Immunol. 190, 3994-4004 (2013).

11. Yoshizaki, M. et al. Spatiotemporal regulation of intracellular trafficking of Toll-like receptor 9 by an inhibitory receptor, Ly49Q. Blood 114, 1518-1527 (2009).

12. Grouard, G. et al. The enigmatic plasmacytoid T cells develop into dendritic cells with interleukin (IL)-3 and CD40-ligand. J. Exp. Med. 185, 1101-1111 (1997).

13. O’Keeffe, M. et al. Mouse plasmacytoid cells: long-lived cells, heterogeneous in surface phenotype and function, that differentiate into CD8(+) dendritic cells only after microbial stimulus. J. Exp. Med. 196, 1307-1319 (2002).

14. Cisse, B. et al. Transcription factor E2-2 is an essential and specific regulator of plasmacytoid dendritic cell development. Cell 135, 37-48 (2008).

15. Ghosh, H. S., Cisse, B., Bunin, A., Lewis, K. L. \& Reizis, B. Continuous expression of the transcription factor e2-2 maintains the cell fate of mature plasmacytoid dendritic cells. Immunity 33, 905-916 (2010).

16. Nagasawa, M., Schmidlin, H., Hazekamp, M. G., Schotte, R. \& Blom, B. Development of human plasmacytoid dendritic cells depends on the combined action of the basic helix-loop-helix factor E2-2 and the Ets factor Spi-B. Eur. J. Immunol. 38, 23892400 (2008).

17. Qin, X. F., An, D. S., Chen, I. S. \& Baltimore, D. Inhibiting HIV-1 infection in human T cells by lentiviral-mediated delivery of small interfering RNA against CCR5. Proc. Natl. Acad. Sci. USA 100, 183-188 (2003).

18. Chaperot, L. et al. Identification of a leukemic counterpart of the plasmacytoid dendritic cells. Blood 97, 3210-3217 (2001).

19. Chaperot, L. et al. Leukemic plasmacytoid dendritic cells share phenotypic and functional features with their normal counterparts. Eur. J. Immunol. 34, 418-426 (2004).

20. Veron, P. et al. Highly efficient transduction of human plasmacytoid dendritic cells without phenotypic and functional maturation. J. Transl. Med. 7, 10 (2009).

21. Sapienza, M. R. et al. Molecular profiling of blastic plasmacytoid dendritic cell neoplasm reveals a unique pattern and suggests selective sensitivity to NF-kB pathway inhibition. Leukemia 28, 1606-1616 (2014).

22. Jahrsdorfer, B. et al. Granzyme B produced by human plasmacytoid dendritic cells suppresses T-cell expansion. Blood 115, 1156-1165 (2010).

23. Huang da, W., Sherman, B. T. \& Lempicki, R. A. Systematic and integrative analysis of large gene lists using DAVID bioinformatics resources. Nat. Protoc. 4, 44-57 (2009).

24. Oosting, M. et al. Human TLR10 is an anti-inflammatory pattern-recognition receptor. Proc. Natl. Acad. Sci. U.S.A. 111, E44784484 (2014).

25. Hasan, U. et al. Human TLR10 is a functional receptor, expressed by B cells and plasmacytoid dendritic cells, which activates gene transcription through MyD88. J. Immunol. 174, 2942-2950 (2005).

26. Angelot-Delettre, F. et al. Intracytoplasmic detection of TCL1--but not ILT7-by flow cytometry is useful for blastic plasmacytoid dendritic cell leukemia diagnosis. Cytometry A. 81, 718-724 (2012)

27. Patel, N. et al. OB-BP1/Siglec-6. a leptin- and sialic acid-binding protein of the immunoglobulin superfamily. J. Biol. Chem. 274, 22729-22738 (1999).

28. Brinkman-Van der Linden, E. C. et al. Human-specific expression of Siglec-6 in the placenta. Glycobiology 17, 922-931 (2007).

29. Kardava, L. et al. Attenuation of HIV-associated human B cell exhaustion by siRNA downregulation of inhibitory receptors. J. Clin. Invest. 121, 2614-2624 (2011).

30. Dijkman, R. et al. Gene-expression profiling and array-based CGH classify CD4+CD56+ hematodermic neoplasm and cutaneous myelomonocytic leukemia as distinct disease entities. Blood 109, 1720-1727 (2007).

31. Dai, L. et al. IL-27 inhibits HIV-1 infection in human macrophages by down-regulating host factor SPTBN1 during monocyte to macrophage differentiation. J. Exp. Med. 210, 517-534 (2013).

32. Sohn, H. W., Krueger, P. D., Davis, R. S. \& Pierce, S. K. FcRL4 acts as an adaptive to innate molecular switch dampening BCR signaling and enhancing TLR signaling. Blood 118, 6332-6341 (2011).

33. Liu, Y. J. IPC: professional type 1 interferon-producing cells and plasmacytoid dendritic cell precursors. Annu. Rev. Immunol. 23, 275-306 (2005).

34. Kamogawa-Schifter, Y. et al. Ly49Q defines 2 pDC subsets in mice. Blood 105, 2787-2792 (2005).

35. Pelayo, R. et al. Derivation of 2 categories of plasmacytoid dendritic cells in murine bone marrow. Blood 105, 4407-4415 (2005).

36. Yang, G. X. et al. CD4- plasmacytoid dendritic cells (pDCs) migrate in lymph nodes by CpG inoculation and represent a potent functional subset of pDCs. J. Immunol. 174, 3197-3203 (2005).

37. Segura, E., Wong, J. \& Villadangos, J. A. Cutting edge: B220+CCR9- dendritic cells are not plasmacytoid dendritic cells but are precursors of conventional dendritic cells. J. Immunol. 183, 1514-1517 (2009).

38. Schlitzer, A. et al. Identification of CCR9- murine plasmacytoid DC precursors with plasticity to differentiate into conventional DCs. Blood 117, 6562-6570 (2011). 
39. Matsui, T. et al. CD2 distinguishes two subsets of human plasmacytoid dendritic cells with distinct phenotype and functions. J. Immunol. 182, 6815-6823 (2009)

40. Zhang, J. Q., Biedermann, B., Nitschke, L. \& Crocker, P. R. The murine inhibitory receptor mSiglec-E is expressed broadly on cells of the innate immune system whereas mSiglec-F is restricted to eosinophils. Eur. J. Immunol. 34, 1175-1184 (2004).

41. Tateno, H., Crocker, P. R. \& Paulson, J. C. Mouse Siglec-F and human Siglec- 8 are functionally convergent paralogs that are selectively expressed on eosinophils and recognize 6'-sulfo-sialyl Lewis X as a preferred glycan ligand. Glycobiology 15, 11251135 (2005).

42. Zhang, M. et al. Defining the in vivo function of Siglec-F, a CD33-related Siglec expressed on mouse eosinophils. Blood 109, 4280-4287 (2007).

43. Chen, G. Y. et al. Broad and direct interaction between TLR and Siglec families of pattern recognition receptors and its regulation by Neu1. Elife 3, e04066 (2014).

\section{Acknowlegements}

This work is supported by National Natural Science Foundation of China (NSFC 31370911) and Beijing Municipal Science \& Technology Commission (SCW 2014-09) to L.Z..

\section{Author Contributions}

M.C. designed and performed experiments, analyzed data and wrote the main manuscript text; X.Z. prepared the critical antibody; H.Y. performed the proliferation experiment; P.D. analyzed the data; J.P. and L.C. provided the cell line GEN2.2; L.S. and L.Z. initiated the study, orgainzed, designed, and wrote the paper.

\section{Additional Information}

Supplementary information accompanies this paper at http://www.nature.com/srep

Competing financial interests: The authors declare no competing financial interests.

How to cite this article: Cheng, M. et al. Characterization of species-specific genes regulated by E2-2 in human plasmacytoid dendritic cells. Sci. Rep. 5, 10752; doi: 10.1038/srep10752 (2015).

(c) (i) This work is licensed under a Creative Commons Attribution 4.0 International License. The images or other third party material in this article are included in the article's Creative Commons license, unless indicated otherwise in the credit line; if the material is not included under the Creative Commons license, users will need to obtain permission from the license holder to reproduce the material. To view a copy of this license, visit http://creativecommons.org/licenses/by/4.0/ 